\title{
On Properties of Hamiltonian Structures for a Class of Evolutionary PDEs
}

\author{
Si-Qi Liu* Chao-Zhong $\mathrm{Wu}^{\dagger}$ Youjin Zhang ${ }^{\ddagger}$ \\ Department of Mathematical Sciences, Tsinghua University, \\ Beijing, P. R. China
}

\begin{abstract}
In [17] it is proved that for certain class of perturbations of the hyperbolic equation $u_{t}=f(u) u_{x}$, there exist changes of coordinate, called quasi-Miura transformations, that reduce the perturbed equations to the unperturbed one. We prove in the present paper that if in addition the perturbed equations possess Hamiltonian structures of certain type, the same quasi-Miura transformations also reduce the Hamiltonian structures to their leading terms. By applying this result, we obtain a criterion of the existence of Hamiltonian structures for a class of scalar evolutionary PDEs and an algorithm to find out the Hamiltonian structures.
\end{abstract}

Key words: Hamiltonian structure, quasi-Miura transformation, quasi-triviality

\section{Introduction}

We consider in this paper the following class of generalized scalar evolutionary PDEs of the unknown function $u=u(x, t)$

$$
u_{t}=f(u) u_{x}+\sum_{k=1}^{\infty} \varepsilon^{k} F_{k}\left(u ; u_{x}, \cdots, u^{(k+1)}\right), \quad f^{\prime}(u) \neq 0,
$$

where $u_{t}=\partial_{t} u, u_{x}=\partial_{x} u, u^{(\ell)}=\partial_{x}^{\ell} u$. The functions $F_{k}$ are assumed to be polynomials of $u_{x}, \ldots, u^{(k+1)}$ with coefficients depending smoothly on $u$, and they are homogeneous of degree $k+1$ under the following assignment of degrees:

$$
\operatorname{deg} u^{(\ell)}=\ell, \ell \geq 1 ; \quad \operatorname{deg} h(u)=0 \text { for a smooth function } h(u) .
$$

\footnotetext{
*liusq@mail.tsinghua.edu.cn

$\dagger$ wucz05@mails.tsinghua.edu.cn

†youjin@mail.tsinghua.edu.cn
} 
Such functions are called homogeneous differential polynomials.

In the case when the right hand side of (1.1) truncates, the equation is a usual evolutionary PDE. In generally, we allow the the right hand side of (1.1) to be nontruncated. Such equations arise, for example, when we consider an evolutionary PDE of the form

$$
u_{t}=K\left(u ; u_{x}, \cdots, u^{(N)}\right)
$$

with a function $K$ being analytic at $u_{x}=\cdots=u^{(N)}=0$ and $K(u ; 0, \cdots, 0)=0$. We can make the rescaling $x \mapsto \varepsilon x, t \mapsto \varepsilon t$, and expand the the right hand side of the equation (1.3) into a power series of $\varepsilon$, the resulting equation is of the form (1.1). Equations of the form (1.1) also appear when we consider PDEs of the following type

$$
u_{t}-u_{x x t}=K\left(u ; u_{x}, \cdots, u^{(N)}\right),
$$

where the function $K$ is given as above. An important example of such class of equations is the Camassa - Holm equation (see Example 3.6 below) which is an integrable nonlinear PDE describing shallow water waves [1, 2, 13]. We can perform the same rescaling and rewrite the equation (1.4) as follows:

$$
u_{t}=\left(1-\varepsilon^{2} \partial_{x}^{2}\right)^{-1} \frac{K\left(u ; \varepsilon u_{x}, \ldots\right)}{\varepsilon}=\sum_{k=0}^{\infty} \varepsilon^{2 k} \partial_{x}^{2 k} \frac{K\left(u ; \varepsilon u_{x}, \ldots\right)}{\varepsilon},
$$

the right hand side of the above equation can also be expanded into the form (1.1). Here the Taylor expansion converges in the formal power series topology.

In this paper we consider properties of the Hamiltonian structures for equations of the form (1.1). Note that equation 1.1) is a perturbation of the hyperbolic equation

$$
v_{t}=f(v) v_{x}, \quad f^{\prime}(v) \neq 0 .
$$

This equation possesses infinitely many Hamiltonian structures. In fact, for any smooth function $g(v)$, there is a Poisson bracket on the space of functionals of $v(x)$ which is defined by

$$
\{v(x), v(y)\}_{g}=g(v) \boldsymbol{\delta}^{\prime}(x-y)+\frac{1}{2} g^{\prime}(v) v_{x} \boldsymbol{\delta}(x-y),
$$

here $v(x), v(y)$ are regarded as distributions. Then equation (1.5) can be expressed as a Hamiltonian system

$$
v_{t}=\left\{v(x), H_{g}\right\}_{g},
$$

where the functional $H_{g}$ is defined by

$$
H_{g}=\int h(v) d x \text {, with } h \text { satisfying } g h^{\prime \prime}+\frac{1}{2} g^{\prime} h^{\prime}=f .
$$


We call the equation (1.1) a Hamiltonian system or a Hamiltonian perturbation of the equation (1.5) if it possesses a Hamiltonian structure

$$
u_{t}=\{u(x), H\},
$$

where the Poisson bracket and the Hamiltonian have the forms

$$
\begin{gathered}
\{u(x), u(y)\}=g(u) \delta^{\prime}(x-y)+\frac{1}{2} g^{\prime}(u) u_{x} \delta(x-y) \\
+\sum_{k=1}^{\infty} \varepsilon^{k} \sum_{l=0}^{k+1} A_{k, l}\left(u ; u_{x}, \cdots, u^{(l)}\right) \delta^{(k+1-l)}(x-y), \\
H=\int\left(h(u)+\sum_{l=1}^{\infty} \varepsilon^{l} B_{l}\left(u ; u_{x}, \cdots, u^{(l)}\right)\right) d x
\end{gathered}
$$

Here $A_{k, l}, B_{l}$ are homogenous differential polynomials of degree $l$.

Studies of Hamiltonian perturbations of general hyperbolic systems were initiated by Dubrovin and the authors of the present paper in [8]. It was proved there that any bihamiltonian perturbation of a hyperbolic system which possesses a semisimple bihamiltonian structure is quasi-trivial, this means that the perturbation terms can be eliminated by a change of the dependent variables, called a quasi-Miura transformation, of the system. In [7], Dubrovin studied the Hamiltonians perturbation of (1.5), and proved certain universality of behavior of solutions of the perturbed equation (1.1) near the point of gradient catastrophe. He also proved that the property of quasi-triviality still holds true at the approximation up to $\varepsilon^{4}$ for Hamiltonian perturbations of [1.5], and it plays an important role in obtaining the main result of [7]. A further study of the quasi-triviality property of equations of the form (1.1) was given in [17], where the following theorem is given on the validity of this property without the assumption of existence of Hamiltonian structures.

Theorem 1.1 (Quasi-Triviality Theorem) For any evolutionary PDE of the form (1.1), there exists a change of the dependent variable called a quasi-Miura transformation

$$
v=u+\sum_{k=1}^{\infty} \varepsilon^{k} \frac{1}{u_{x}^{L_{k}}} \sum_{m=0}^{M_{k}} Y_{k, m}\left(u ; u_{x}, \cdots, u^{\left(N_{k}\right)}\right)\left(\log u_{x}\right)^{m}
$$

which reduces the equation (1.1) to its leading term (1.5). Here $L_{k}, M_{k}, N_{k}$ are certain integers that only depend on $k$, and $Y_{k, m}$ are homogenous differential polynomials of degree $L_{k}+k$.

The quasi-Miura transformation of the above theorem is also called the reducing transformation for (1.1). In [17] this theorem is also applied to obtain a criterion of integrability for the class of equations of the form (1.1). 
Now given a Hamiltonian perturbation (1.1), we consider in this paper the relationship between the Hamiltonian structure and its reducing transformation. The main result is the following:

Theorem 1.2 (Main Theorem) For any Hamiltonian perturbation (1.1) of the equation (1.5) with a Hamiltonian structure of the form (1.8), (1.9), the reducing transformation (1.10) does not depend on $\log u_{x}$, and it also reduces the Hamiltonian structure (1.8), (1.9) to its leading term (1.6), (1.7).

By inverting the quasi-Miura transformation, the theorem implies that any Hamiltonian structure of (1.1) comes from a Hamiltonian structure of the form (1.5). This fact provides a method to determine whether an equation of the form 1.1) possesses Hamiltonian structures, and if so how to find them.

The paper is organized as follows: in the next section, we give a proof of the main theorem; in Section 3, we explain in detail the application of the Main Theorem to determine the existence of Hamiltonian structures for evolutionary PDEs of the form (1.1), and to find out the Hamiltonian structures. In the final section we give some concluding remarks.

\section{Proof of the main theorem}

We first recall some useful notations from [17]. Denote $u_{\ell}=u^{(\ell)}(\ell=0,1,2, \cdots)$, and define the ring of differential polynomials by $\mathscr{R}=C^{\infty}\left(u_{0}\right)\left[u_{1}, u_{2}, \cdots\right]$. It is a graded ring with degrees given by (1.2). Let $\mathscr{A}$ be its formal completion

$$
\mathscr{A}=\left\{f=\sum_{i=0}^{\infty} \varepsilon^{i} f_{i} \mid f_{i} \in \mathscr{R}, \operatorname{deg} f_{i}=i\right\} .
$$

Elements of $\mathscr{A}$ are also called differential polynomials.

For any $X \in \mathscr{A}$, we can define a derivation on $\mathscr{A}$

$$
\hat{X}=\sum_{i=0}^{\infty} \partial_{x}^{i} X \frac{\partial}{\partial u_{i}}
$$

The map $\mathscr{A} \rightarrow \operatorname{Der} \mathscr{A}, X \mapsto \hat{X}$ induces the following Lie bracket on $\mathscr{A}$ :

$$
[X, Y]=\hat{X}(Y)-\hat{Y}(X), \forall X, Y \in \mathscr{A},
$$

and $\mathscr{A}$ becomes a module of this Lie algebra. The Lie algebra $\mathscr{A}$ contains a subalgebra

$$
\mathscr{B}=\left\{f=\sum_{i=1}^{\infty} \varepsilon^{i} f_{i} \mid f_{i} \in \mathscr{R}, \operatorname{deg} f_{i}=i\right\},
$$


each element $X \in \mathscr{B}$ defines an evolutionary PDE of the form (1.1)

$$
u_{t}=\varepsilon^{-1} X=f(u) u_{x}+\varepsilon f_{2}+\varepsilon^{2} f_{3}+\cdots,
$$

(without the assumption $f^{\prime}(u) \neq 0$ in general), so we will also call $X$ an evolutionary PDE. If in additional $f^{\prime}(u) \neq 0$, we say that the above PDE is generic. Let $X \in \mathscr{B}$ be an evolutionary PDE, $h \in \mathscr{A}$ be a differential polynomial. If there exists a $\sigma \in \mathscr{A}$ such that $\hat{X}(h)=\partial_{x} \sigma$, then $h$ is called a conserved density of $X$.

Consider the quotient space

$$
\Lambda=\mathscr{A} /\left(\partial_{x} \mathscr{A} \oplus \mathbb{R}\right)
$$

the coset corresponding to $f \in \mathscr{A}$ is denoted by $\int f d x$, it is called a local functional defined by the density $f$. Note that $\int \cdot d x$ is just a formal notation, it doesn't mean integration here. It's easy to see that $\Lambda$ is also an $\mathscr{A}$-module (as a module of Lie algebra), the action of $X$ on $F=\int f d x$ is given in the following natural way:

$$
X . F=\int \hat{X}(f) d x=\int X \frac{\delta F}{\delta u} d x
$$

where

$$
\frac{\delta F}{\delta u}=\sum_{i=0}^{\infty}\left(-\partial_{x}\right)^{i} \frac{\partial f}{\partial u^{i}}
$$

is the variational derivative. In terms of these notations, $h \in \mathscr{A}$ is a conserved density of $X$ if and only if the local functional $H=\int h d x$ satisfies $X . H=0$. Local functionals of this type are called the conserved quantities of $X$.

Let us introduce the algebra of differential operators on $\mathscr{A}$ as follows:

$$
\mathscr{D}=\left\{P=\sum_{k=0}^{\infty} \varepsilon^{k} \sum_{s=0}^{k+1} P_{k, s} \partial_{x}^{k+1-s} \mid P_{k, s} \in \mathscr{R}, \operatorname{deg} P_{k, s}=s\right\} .
$$

It is a graded algebra under the definition (1.2) and $\operatorname{deg} \partial_{x}=1$. A differential operator $P \in \mathscr{D}$ is called a Hamiltonian operator if the bilinear operation

$$
\{\cdot, \cdot\}_{P}: \Lambda \times \Lambda \rightarrow \Lambda, \quad(F, G) \mapsto \int \frac{\delta F}{\delta u} P\left(\frac{\delta G}{\delta u}\right) d x
$$

forms a Lie bracket. Note that every Hamiltonian structure of the form (1.8) corresponds to a unique Hamiltonian operator

$$
P=g(u) \partial_{x}+\frac{1}{2} g^{\prime}(u) u_{x}+\sum_{k=1}^{\infty} \varepsilon^{k} \sum_{l=0}^{k+1} A_{k, l}\left(u ; u_{x}, \cdots, u^{(l)}\right) \partial_{x}^{k+1-l}
$$

and vice versa. 
Example 2.1 Let $P$ be a Hamiltonian operator of the form

$$
P=P_{1}+\varepsilon^{k-1} P_{k}+O\left(\varepsilon^{k}\right), \quad k \geq 2,
$$

it is easy to see that the leading term $P_{1}=P_{10} \partial_{x}+P_{11}$ is also a Hamiltonian operator. The skew-symmetry condition of the Lie bracket (2.3) implies that $P_{1}$ must be of the form

$$
P_{1}=\phi(u) \partial_{x}+\frac{1}{2} \phi^{\prime}(u) u_{x}
$$

One can verify that a differential operator of the form (2.4) is automatically a Hamiltonian operator. The Jacobi identity for $\{\cdot, \cdot\}_{P}$ implies that the equality

$$
\begin{aligned}
& \left\{\{F, G\}_{P_{k}}, H\right\}_{P_{1}}+\left\{\{F, G\}_{P_{1}}, H\right\}_{P_{k}}+\left\{\{G, H\}_{P_{k}}, F\right\}_{P_{1}}+ \\
& \left\{\{G, H\}_{P_{1}}, F\right\}_{P_{k}}+\left\{\{H, F\}_{P_{k}}, G\right\}_{P_{1}}+\left\{\{H, F\}_{P_{1}}, G\right\}_{P_{k}}=0
\end{aligned}
$$

holds true for any local functionals $F, G, H \in \Lambda$. The differential operator $\varepsilon^{k-1} P_{k}$ satisfying the above condition is called an infinitesimal deformation of the Hamiltonian operator $P_{1}$.

Definition 2.2 An evolutionary PDE $X \in \mathscr{B}$ is called to be Hamiltonian if there exists a Hamiltonian operator $P$ and a local functional $H$ such that

$$
X=P . H=P\left(\frac{\delta H}{\delta u}\right) .
$$

This $X$ is also called a Hamiltonian vector field with respect to the Hamiltonian operator $P$, and $H$ is called the Hamiltonian of $X$.

Proposition 2.3 For any evolutionary $P D E X \in \mathscr{B}$ and differential operator $P \in \mathscr{D}$, there exists a unique differential operator $Q \in \mathscr{D}$ such that for any local functionals $F, G$ we have

$$
\{F, G\}_{Q}=X .\{F, G\}_{P}-\{X . F, G\}_{P}-\{F, X . G\}_{P},
$$

The operator $Q$ is called the Lie derivative of $P$ w.r.t. $X$, and is denoted by $\operatorname{Lie}_{X} P$.

Proof In fact, suppose $P=\sum_{s=0}^{\infty} P_{s} \partial_{x}^{s}$, we have

$$
Q=\sum_{s=0}^{\infty} \hat{X}\left(P_{s}\right) \cdot \partial_{x}^{s}-\sum_{k=0}^{\infty}\left(\frac{\partial X}{\partial u_{k}} \cdot \partial_{x}^{k} \cdot P+P \cdot\left(-\partial_{x}\right)^{k} \cdot \frac{\partial X}{\partial u_{k}}\right),
$$

where $\cdot$ is the multiplication in $\mathscr{D}$. The proposition is proved.

The elements of $\Lambda, \mathscr{A}, \mathscr{D}$ are examples of local 0-, 1-, 2- vectors. In general, we can define the space of local $k$-vectors $\Lambda_{l o c}^{k}$ and the Schouten-Nijenhuis bracket between them

$$
[\cdot, \cdot]: \Lambda_{l o c}^{k} \times \Lambda_{l o c}^{l} \rightarrow \Lambda_{l o c}^{k+l-1}
$$


The formulae (2.2), (2.5), (2.6) are just particular cases of the Schouten-Nijenhuis bracket, in fact we have the following correspondence:

$$
X . F=[X, F], \quad P . H=[P, H], \quad \operatorname{Lie}_{X} P=[X, P] .
$$

The Schouten-Nijenhuis bracket satisfies the following identities

$$
\begin{aligned}
& {[P, Q]=(-1)^{p q}[Q, P],} \\
& (-1)^{p r}[[P, Q], R]+(-1)^{q p}[[Q, R], P]+(-1)^{r q}[[R, P], Q]=0,
\end{aligned}
$$

where $P \in \Lambda_{l o c}^{p}, Q \in \Lambda_{l o c}^{q}, R \in \Lambda_{l o c}^{r}$. We omit the general definition of $\Lambda_{l o c}^{k}$ and the Schouten-Nijenhuis bracket here, for more details one can refer to [9, 16, 8].

Definition 2.4 A Hamiltonian operator $P$ is called of hydrodynamic type if

$$
P=g(u) \partial_{x}+\frac{1}{2} g^{\prime}(u) u_{x}, g(u) \neq 0
$$

Hamiltonian operators with hydrodynamic leading terms are called generic.

Theorem 2.5 [5] 14] Let $P$ be a Hamiltonian operator of hydrodynamic type.

(a) If $Q \in \mathscr{D}$ is an infinitesimal deformation of $P$, then there exists $X \in \mathscr{B}$ such that $Q=\mathrm{Lie}_{X} P$;

(b) If $X \in \mathscr{B}$ satisfies $\operatorname{Lie}_{X} P=0$, then there exists a local functional $H \in \Lambda$ such that $X=P . H$.

This theorem is implied by the vanishing of the Poisson cohomologies [15] of the Hamiltonian structure (2.9) as proved in [14](c.f. [10]), see also [5, 9].

The identity (2.8) implies that for any $X \in \mathscr{B}$, the map

$$
g_{X}: \Lambda_{l o c}^{*} \rightarrow \Lambda_{l o c}^{*}, K \mapsto e^{\mathrm{ad}_{X}} K=\sum_{n=0}^{\infty} \frac{1}{n !}\left(\operatorname{ad}_{X}\right)^{n} K
$$

is an automorphism of the graded Lie algebra $\left(\Lambda_{l o c}^{*},[\cdot, \cdot]\right)$. Such an automorphism is called a Miura type transformation.

Corollary 2.6 [5] 9 , 14] For any generic Hamiltonian operator $P$ with leading term $P_{1}$, there exists a Miura type transformation that reduces $P$ to its leading term $P_{1}$. In other words, there exist an $X \in \mathscr{B}$ such that $g_{X}(P)=P_{1}$.

Lemma 2.7 Let $X \in \mathscr{B}$ be a Hamiltonian vector field with respect to a generic Hamiltonian operator $P$, and

$$
\varepsilon^{-1} X=f(u) u_{x}+\varepsilon X_{2}+\varepsilon^{2} X_{3}+\cdots, P=P_{1}+\varepsilon P_{2}+\varepsilon^{2} P_{3}+\cdots
$$

then the coefficients $X_{2}$ and $P_{2}$ of $\varepsilon$ must vanish. 
Proof By using Theorem 2.5 we know the existence of $Y=h(u) u_{x} \in \mathscr{B}$ such that $P_{2}=\operatorname{Lie}_{Y} P_{1}$, then by using the formula (2.6) we easily obtain $P_{2}=0$. Let $H=$ $H_{0}+\varepsilon H_{1}+\cdots$ be the Hamiltonian of $X$. From the definition of local functionals we see that $H_{1}$ always vanishes, so $X_{2}=\left[P_{1}, H_{1}\right]+\left[P_{2}, H_{0}\right]=0$. The lemma is proved.

Now let us consider the quasi-triviality of evolutionary PDEs. Define

$$
\tilde{\mathscr{R}}=\left(\mathscr{R}\left[u_{1}^{-1}\right]\right)_{\geq 0}=C^{\infty}\left(u_{0}\right)\left[u_{1}, \frac{u_{2}}{u_{1}^{2}}, \frac{u_{3}}{u_{1}^{3}}, \cdots\right],
$$

whose elements are called almost differential polynomials [8]. We can modify the above definition of the spaces $\mathscr{A}, \mathscr{B}, \Lambda, \mathscr{D}$ by replacing $\mathscr{R}$ with $\tilde{\mathscr{R}}$, and denote the resulting spaces by $\tilde{\mathscr{A}}, \tilde{\mathscr{B}}, \tilde{\Lambda}, \tilde{\mathscr{D}}$. The Schouten-Nijenhuis brackets (2.2), (2.5), (2.6) are defined in the same way.

Lemma 2.8 Let $X \in \mathscr{B}$ be an evolutionary PDE of the following form

$$
X=\varepsilon f(u) u_{x}+\sum_{i \geq 3} \varepsilon^{i} X_{i}, X_{i} \in \mathscr{R}, \operatorname{deg} X_{i}=i,
$$

then there exists a unique $Y \in \tilde{B}$ of the form

$$
Y=\sum_{i \geq 2} \varepsilon^{i} Y_{i}, Y_{i} \in \tilde{\mathscr{R}}, \operatorname{deg} Y_{i}=i,
$$

such that $g_{Y}(X)=\varepsilon f(u) u_{x}$. Any other reducing transformation $g_{Z}(Z \in \tilde{\mathscr{B}})$ of $X$ can be represented as $g_{\tilde{Y}} \circ g_{Y}$, where $\tilde{Y}=\varepsilon p(u) u_{x}$ and $p(u)$ is an arbitrary smooth function.

Proof This is a weaker version of the quasi-triviality theorem. The proof is similar to the full version, see [17].

Lemma 2.9 Let $X=\varepsilon f(u) u_{x}, f^{\prime}(u) \neq 0$. If a homogeneous differential operator $P \in \tilde{\mathscr{D}}$ with $\operatorname{deg} P \geq 2$ satisfies $\operatorname{Lie}_{X} P=0$, then $P=0$.

Proof We only need to consider the case when $f(u)=u$, since other cases can be converted to this case by applying a change of coordinate $\tilde{u}=f(u)$. Let the highest order of $\partial_{x}$ in $P$ be $m$, then

$$
P=P_{m} \partial_{x}^{m}+\cdots, P_{m} \neq 0, \operatorname{deg} P_{m}=\operatorname{deg} P-m \geq 2-m .
$$

The vanishing of the coefficient of $\partial_{x}^{m}$ in $\mathrm{Lie}_{X} P$ implies

$$
\sum_{k=0}^{N}\left(\partial_{x}^{k}\left(u u_{1}\right)-u u_{k+1}\right) \frac{\partial P_{m}}{\partial u_{k}}+(m-1) u_{1} P_{m}=0 .
$$


Here $N$ is the biggest indices of $u_{k}$ that $P_{m}$ depends on. By using Lemma 4.1 of [17], the general solution to the above equation reads

$$
P_{m}=u_{x}^{1-m} c\left(u, \phi_{2}, \phi_{3}, \cdots, \phi_{N}\right),
$$

where $c$ is an arbitrary smooth function, and

$$
\phi_{1}=\frac{1}{u_{1}}, \phi_{k+1}=\frac{1}{u_{1}} \sum_{i=1}^{k} u_{i+1} \frac{\partial \phi_{k}}{\partial u_{i}}, k=1,2, \cdots .
$$

Note that $P_{m}$ depends polynomially on $u_{2}, u_{3}, \cdots$, it follows from the above definition of the functions $\phi_{k}$ that $c$ also depends polynomially on $\phi_{2}, \ldots, \phi_{N}$. From the fact that $\operatorname{deg} \phi_{k}=-1$ we obtain $\operatorname{deg} P_{m}=1-m+\operatorname{deg} c \leq 1-m$, this contradicts with (2.12), so we must have $P=0$. The lemma is proved.

Corollary 2.10 The Hamiltonian operator of a generic evolutionary PDE is also generic.

Proof Let $X=\varepsilon f(u) u_{x}+\cdots$ be a generic evolution PDE, $P=\varepsilon^{m-1} P_{m}+\cdots, P_{m} \neq 0$ be a Hamiltonian operator of $X$, then we have $\operatorname{Lie}_{f(u) u_{x}} P_{m}=0$. If $m \geq 2$, then by the above lemma we have $P_{m}=0$, which contradicts with our assumption $P_{m} \neq 0$, so we have $m=1$. The corollary is proved.

Lemma 2.11 Let $P$ be a Hamiltonian operator of hydrodynamic type, $H \in \tilde{\Lambda}$ be homogeneous and $\operatorname{deg} H \geq 1$. If P. $H=0$, then $H=0$.

Proof We denote the density of $H$ by $h=F\left(u, u_{x}, \cdots, u_{N}\right), N \geq 1$. Let $X=P . H$, then we have

$$
\frac{\partial X}{\partial u_{2 N+1}}=(-1)^{N} g(u) \frac{\partial^{2} F}{\partial u_{N}^{2}}=0 .
$$

So we have $h=F_{1}\left(u, u_{x}, \cdots, u_{N-1}\right) u_{N}+F_{0}\left(u, u_{x}, \cdots, u_{N-1}\right)$. After integrating by part, we can assume that the density of $H$ does not depend on $u_{N}$. By induction on $N$, we know that the density of $H$ can be chosen to depend only on $u$. However, by assumption we have $\operatorname{deg} H \geq 1$, so we must have $H=0$. The lemma is proved.

Proof of the Main Theorem: Let $X \in \mathscr{B}$ be a generic evoutionary PDE

$$
X=\varepsilon X_{1}+\varepsilon^{2} X_{2}+\cdots, \quad X_{1}=f(u) u_{x}, f^{\prime}(u) \neq 0
$$

with a Hamiltonian structure $X=P . H$, where

$$
P=P_{1}+\varepsilon P_{2}+\cdots \in D, \quad H=H_{0}+\varepsilon H_{1}+\cdots \in \Lambda .
$$

It follows from Lemma 2.7 and 2.8 that there exists $Y \in \tilde{\mathscr{B}}$ such that

$$
g_{Y}(X)=\varepsilon X_{1}=\varepsilon f(u) u_{x} .
$$


Since $g_{Y}$ is an automorphim, we have

$$
\operatorname{Lie}_{\varepsilon f(u) u_{x}} g_{Y}(P)=g_{Y}\left(\operatorname{Lie}_{X} P\right)=0 .
$$

By using Lemma 2.9 we arrive at $g_{Y}(P)=P_{1}$.

By using the fact that $g_{Y}$ is an automorphim again, we get

$$
\varepsilon f(u) u_{x}=g_{Y}([P, H])=\left[P_{1}, g_{Y}(H)\right],
$$

so it follows from Lemma2.11 that $g_{Y}(H)=H_{0}$. The theorem is proved.

\section{The Hamiltonian perturbations}

In this section we propose a way to determine whether a given generic evolutionary PDE is Hamiltonian, and if so, how to find its Hamiltonian structures.

Lemma 2.7 shows that a generic Hamiltonian PDE in $\mathscr{B}$ must take the form

$$
X=\varepsilon f(u) u_{x}+\sum_{i \geq 3} \varepsilon^{i} X_{i}, \quad f^{\prime}(u) \neq 0, X_{i} \in \mathscr{R}, \operatorname{deg} X_{i}=i .
$$

Moreover, the associated Hamiltonian structure $P$ must be generic, i.e. its leading term has the form (2.4) with $\phi(u) \neq 0$. According to the Main Theorem, the reducing transformation $g_{Y}$ satisfying $g_{Y}(X)=\varepsilon f(u) u_{x}$ also reduces $P$ to its leading term $P_{1}$, where $Y$ is of the form (2.11) given in Lemma2.8. Thus we have

$$
P=g_{Y}^{-1} P_{1}=e^{-\operatorname{ad}_{Y}}\left(\phi(u) \partial_{x}+\frac{1}{2} \phi^{\prime}(u) u_{x}\right)
$$

and the following corollary of the Main Theorem:

Corollary 3.1 A given generic PDE of the form (3.1) possesses a Hamiltonian structure of the form (1.8) if and only if there exists a non-vanishing smooth function $\phi(u)$ such that the bivector (3.2) lies in $\mathscr{D}$.

Given a generic $X$ of the form 3.1), its reducing transformation $g_{Y}$ can be found explicitly by using the method given in [17]. So the right hand side of (3.2) can be computed straightforwardly. In this way we obtain a criterion for testing whether a generic PDE is Hamiltonian or not. This algorithm is not hard to be carried out with the help of a computer program, and we give some examples below to illustrate it.

Example 3.2 We consider generic evolutionary PDEs of the form

$$
u_{t}=u u_{x}+\varepsilon^{2}\left(b(u) u_{x x x}+c(u) u_{x x} u_{x}+d(u) u_{x}^{3}\right),
$$

where $b(u), c(u), d(u)$ are smooth functions of $u$, and $b(u) \neq 0$. We are to determine under what conditions such a system is Hamiltonian. 
Lemma 3.3 If the equation (3.3) possesses a Hamiltonian structure with leading term (2.4), then the functions $b(u), c(u), d(u)$ must satisfy

$$
b^{2} \phi^{\prime \prime}-5 b b^{\prime} \phi^{\prime}+\left(\frac{4}{3} c^{2}-16 b d-\frac{20}{3} c b^{\prime}+8 b^{\prime 2}+6 b c^{\prime}-4 b b^{\prime \prime}\right) \phi=0 .
$$

Proof The reducing transformation of the equation 3.3 can be taken to depend only on even powers of $\varepsilon$. Thus the Hamiltonian structure has the form $P=g_{Y}^{-1} P_{1}=$ $P_{1}+\varepsilon^{2} Z_{1}+\varepsilon^{4} Z_{2}+\varepsilon^{6} Z_{3}+\varepsilon^{8} Z_{4}+\cdots$. A direct calculation shows that the term $Z_{1}$ is always a polynomial, and the polynomiality of $Z_{2}$ implies (3.4).

The polynomiality of $Z_{3}, Z_{4}, \cdots$ leads to a set of ODEs of $b(u), c(u), \phi(u)$. These ODEs are too complicated to solve. Here we add a homogeneity condition to simplify the problem (see [17] for a similar condition). Since $b(u) \neq 0, \phi(u) \neq 0$, without loss of generality we can assume that

$$
b(u)=u^{1-2 \alpha}, c(u)=\beta u^{-2 \alpha}, \phi(u)=u^{\gamma}
$$

where $\alpha, \beta, \gamma \in \mathbb{R}$. It follows immediately from equation (3.4) that

$$
d(u)=\frac{u^{-1-2 \alpha}}{48}\left(24-72 \alpha+48 \alpha^{2}-20 \beta+4 \alpha \beta+4 \beta^{2}-18 \gamma+30 \alpha \gamma+3 \gamma^{2}\right) .
$$

Proposition 3.4 The equation (3.3) with $b(u), c(u), d(u)$ given by (3.5), (3.6) possesses a Hamiltonian structure if and only if the constants $\alpha, \beta, \gamma$ satisfy one of the following conditions:

$$
\begin{aligned}
\text { i) } & 8 \alpha+2 \beta+3 \gamma-4=0 \\
\text { ii) } & \alpha=0,2 \beta+3 \gamma-6=0 \\
\text { iii) } & \beta=0,2 \alpha+\gamma-2=0 \\
\text { iv) } & (\alpha, \beta, \gamma)=\left(\frac{1}{2},-\frac{3}{2}, 0\right),\left(\frac{1}{2},-3,0\right),\left(\frac{1}{2},-3,1\right),\left(0,-1, \frac{10}{3}\right) .
\end{aligned}
$$

Proof By solving a system of algebraic equations, we can obtain conditions i)-iv) from the polynomiality of $Z_{3}, Z_{4}$. So these conditions are necessary.

Now denote by $\varepsilon^{-1} X_{1}, \varepsilon^{-1} X_{2}, \varepsilon^{-1} X_{3}$ the right hand side of (3.3) such that $\alpha, \beta, \gamma$ satisfy the condition i), ii), iii) respectively. We define the following differential operators:

$$
\begin{aligned}
& P_{1}=u^{\frac{\gamma}{2}} \partial_{x} u^{\frac{\gamma}{2}} \\
& P_{2}=u^{\frac{\gamma}{2}}\left(\partial_{x}+\varepsilon^{2} \partial_{x}^{3}\right) u^{\frac{\gamma}{2}} \\
& P_{3}=u^{1-2 \alpha}\left(u^{2 \alpha} \partial_{x}+\alpha u^{2 \alpha-1} u_{x}+(\alpha+1) \varepsilon^{2} \partial_{x}^{3}\right) u^{1-2 \alpha}
\end{aligned}
$$


Then by a straightforward computation, we can show that $P_{i}$ are Hamiltonian operators and $\operatorname{Lie}_{X_{i}} P_{i}=0$, i.e. $P_{i}$ is a Hamiltonian structure for $X_{i}$.

In the other four exceptional cases, the equation 3.3 possesses bihamiltonian structures, as it will be shown by the next proposition. The proposition is proved.

An evolutionary PDE of the form (1.1) that is given by a vector field $X \in \mathscr{B}$ is called bihamiltonian, or a bihamiltonian perturbation of (1.5), if there are two compatible Hamiltonian operators $P, Q$ and two local functionals $H_{1}, H_{2}$ such that $X=P . H_{1}=Q \cdot H_{2}$. Here the word "compatible" means that any linear combination of $P, Q$ is also a Hamiltonian operator.

From the Main Theorem it follows that the reducing transformation of the bihamiltonian equation reduces both Hamiltonian operators $P$ and $Q$ to their leading terms which have the form (2.9). It is easy to see that any two Hamiltonian operators of the form (2.9) are compatible, so the two Hamiltonian operators $P, Q$ of a bihamiltonian equation (1.1) are automatically compatible.

Proposition 3.5 The equation (3.3) with $b(u), c(u), d(u)$ given by (3.5), (3.6) possesses a bihamiltonian structure if and only if the constants $(\alpha, \beta)$ are given by one of the following pairs:

$$
\left(\frac{1}{2}, 0\right),\left(\frac{1}{2},-\frac{3}{2}\right),\left(\frac{1}{2},-3\right),(0,-2),(0,-1) .
$$

Proof For an equation (3.3) determined by the parameters $\alpha, \beta$, the bihamiltonian condition requires that there exist two distinct $\gamma_{1}, \gamma_{2}$ such that each $\left(\alpha, \beta, \gamma_{1}\right),\left(\alpha, \beta, \gamma_{2}\right)$ satisfies one of the conditions 3.7-3.10). It's easy to check the pairs of $\alpha, \beta$ given in (3.14) are the only ones that meet the above requirement. Note that for all these cases, the equation (3.3) has been analysed in [17]. The first three cases are equivalent to the $\mathrm{KdV}$ equation via Miura-type transformations, so they possess bihamiltonian structures which are induced from that of the KdV equation via the Miura-type transformations.

For the case when $(\alpha, \beta)=(0,-2)$, we have $\gamma=\frac{8}{3}, \frac{10}{3}$, which are implied by the conditions (3.7) and (3.8). So the corresponding equation has the bihamiltonian structure given by (3.11) and (3.12).

For the case when $(\alpha, \beta)=(0,-1)$, we have $\gamma=\frac{8}{3}, \frac{10}{3}$. The first Hamiltonian structure is given by $(3.12)$, and the second one is given by $P_{2}=u^{\frac{4}{3}} Q u^{\frac{4}{3}}$, where the differential operator $Q$ reads

$$
\begin{aligned}
Q= & w^{2} \partial_{x}+w w_{1} \\
& +\varepsilon^{2}\left(2 w^{2} \partial_{x}^{3}+6 w w_{1} \partial_{x}^{2}+4 w_{1}^{2} \partial_{x}+4 w w_{2} \partial_{x}+3 w_{1} w_{2}+w w_{3}\right) \\
& +\varepsilon^{4}\left(w^{2} \partial_{x}^{5}+5 w w_{1} \partial_{x}^{4}+4 w_{1}^{2} \partial_{x}^{3}+4 w w_{2} \partial_{x}^{3}+3 w_{1} w_{2} \partial_{x}^{2}+w w_{3} \partial_{x}^{2}\right),
\end{aligned}
$$

and $w=u^{\frac{1}{3}}$. The proposition is proved. 
Example 3.6 Consider the equation

$$
u_{t}-\varepsilon^{2} u_{x x t}=u u_{x}+\varepsilon^{2}\left(b u u_{x x x}+c u_{x x} u_{x}\right),
$$

where $b, c \in \mathbb{R}$. When $b=-\frac{1}{3}, c=-\frac{2}{3}$ this equation is called the Camassa-Holm equation, it is an integrable system that was derived as a shallow water wave equation in [1] 2], this system also appeared in the study of hereditary symmetries of soliton equations in [13].

Note that if $(b, c)=(-1,-3)$, the equation is equivalent to its leading term $u_{t}=$ $u u_{x}$. So we assume $(b, c) \neq(-1,-3)$.

Lemma 3.7 If the equation (3.15) possesses a Hamiltonian structure with leading term (2.4), The function $\phi(u)$ must have the form $\phi(u)=\gamma u^{d}$.

Proof As in the above example, we can expand the right hand side of (3.2) in the form $P_{1}+\sum_{k \geq 1} \varepsilon^{2 k} Z_{k}$. The condition that $Z_{2}$ and $Z_{3}$ are differential polynomials yields two ODEs of $\phi(u)$ whose solutions must be power functions.

Without loss of generality, we assume that $\gamma=1$, i.e. $\phi(u)=u^{d}$.

Proposition 3.8 The equation (3.15) possesses a Hamiltonian structure if and only if the constants $(b, c, d)$ satisfy one of the following conditions

$$
\begin{aligned}
\text { i) } \quad d=0, c=2 b \\
\text { ii) } \quad d=0, c=3 b \\
\text { iii) } \quad(b, c, d)=\left(-\frac{1}{3},-\frac{2}{3}, 1\right) \\
\text { iv) } \quad(b, c, d)=(0,-1,2) .
\end{aligned}
$$

Proof The proof is similar to that of the previous example. The four Hamiltonian operators under the coordinate $\tilde{u}=u-\varepsilon^{2} u_{x x}$ read

$$
\begin{aligned}
& P_{1}=\partial_{x}-\varepsilon^{2} \partial_{x}^{3} ; \\
& P_{2}=\left(1+b \varepsilon^{2} \partial_{x}^{2}\right)\left(\partial_{x}-\varepsilon^{2} \partial_{x}^{3}\right) ; \\
& P_{3}=\tilde{u} \partial_{x}+\frac{1}{2} \tilde{u}_{x} ; \\
& P_{4}=\tilde{u}\left(\sum_{k=0}^{\infty} \varepsilon^{2 k} \partial_{x}^{2 k+1}\right) \tilde{u} .
\end{aligned}
$$

The proposition is proved.

Corollary 3.9 The Camassa-Holm equation is the only one of the form (3.15) that possesses a bihamiltonian structure. 
Remark 3.10 By using the method introduced in [17], we can prove that the equation (3.15) is formally integrable if and only if the constants $(b, c)$ is given by one of the following pairs:

$$
\begin{aligned}
& (-1,-2),\left(-1, \frac{1}{2}\right),\left(-1,-\frac{1}{2}\right),\left(-\frac{1}{3},-1\right), \\
& \left(-\frac{1}{3},-\frac{2}{3}\right),\left(-\frac{1}{4},-\frac{3}{4}\right),\left(-\frac{1}{4},-\frac{1}{2}\right) .
\end{aligned}
$$

Here formal integrability of an evolutionary PDE of the form (1.1) means, in the sense of [17], that any symmetry of the equation 1.5] can be extended to a symmetry of (1.1). Note that the pair $\left(-\frac{1}{3},-\frac{2}{3}\right)$ corresponds to the Camassa-Holm equation, which is bihamiltonian; the pair $\left(-\frac{1}{4},-\frac{3}{4}\right)$ corresponds to the Degasperis-Procesi equation [3] 4] which is formally integrable but has only one local Hamiltonian structure.

Example 3.11 According to the Drinfeld-Sokolov construction [6], The Kac-Moody algebra $A_{2}^{(2)}$ is associated to the following evolutionary PDEs:

$$
\begin{aligned}
& u_{t_{1}}=20 u^{2} u_{x}+\varepsilon^{2}\left(10 u u_{x x x}+25 u_{x x} u_{x}\right)+\varepsilon^{4} u_{x x x x x}, \\
& u_{t_{2}}=\frac{5}{4} u^{2} u_{x}+\varepsilon^{2}\left(\frac{5}{2} u u_{x x x}+\frac{5}{2} u_{x x} u_{x}\right)+\varepsilon^{4} u_{x x x x x} .
\end{aligned}
$$

They have the Hamiltonian structure

$$
\frac{\partial u}{\partial t_{i}}=\left(\varepsilon^{2} \partial^{3}+2 u \partial+u_{x}\right) \frac{\delta H_{i}}{\delta u}, \quad i=1,2 .
$$

Here the Hamiltonian $H$ are given respectively by

$$
H_{1}=\int\left(\frac{4}{3} u^{3}-\frac{1}{2} u_{x}^{2}\right) d x, \quad H_{2}=\int\left(\frac{1}{12} u^{3}-\frac{1}{2} u_{x}^{2}\right) d x .
$$

The above algorithm can be carried out to show that the above two equations have no other local Hamiltonian structures.

\section{Concluding remarks}

We showed that the reducing transformation of a Hamiltonian perturbation 1.10 of the hyperbolic equation (1.5) also reduces its Hamiltonian structures to their leading terms. We also applied this fact to the study of existence of Hamiltonian structures for a generic evolutionary PDE of the form (1.1). Note that the derivation of the these results relies on the fact that the evolutionary PDEs we are considering are scalar. It is interesting to generalize these results to evolutionary PDEs that are perturbations 
of certain multi-component hyperbolic systems, such generalization is important in particular in the study of integrability for certain nonlinear evolutionary PDEs. We finish the paper with the following remarks:

Remark 4.1 Let us note that the bihamiltonian equations which appear in Examples 3.2 and 3.6 are also formally integrable in the sense of [17], i.e. any symmetry of their leading terms can be extended to a symmetry of themselves. By combining the main results of [16], [17] and the present paper, we can prove this fact for any evolutionary PDEs of the form [1.1]. Furthermore, the main results of [8] and [16] imply the following theorem:

Theorem 4.2 Let $(P, Q)$ be a semisimple bihamiltonian structure of hydrodynamic type with leading term $\left(P_{1}, Q_{1}\right)$. Then any bihamiltonian vector field of $\left(P_{1}, Q_{1}\right)$ can be extended to a bihamiltonian vector field of $(P, Q)$.

We note that the semisimplicity condition is automatically satisfied in the scalar case.

Remark 4.3 The quasi-Miura transformation (1.10) that reduces the equation (1.1) to its leading term (1.5) is understood as a formal power series in $\varepsilon$. When (1.1) is a Hamiltonian perturbation, it is conjectured by Dubrovin in $[7]$ that for any smooth solution $v(x, t)$ to (1.5) defined for all $x \in \mathbb{R}$ and $0 \leq t<t_{0}$ which is monotone in $x$ for any $t$, the associated reducing transformation gives the asymptotic expansion at $\varepsilon \rightarrow 0$ of a solution $u=u(x, t ; \varepsilon)$ to (1.1) defined on the same domain in the $(x, t)$ plane.

Note that for some nonlinear PDEs that are covariant under the action of certain Lie groups, Flato and his collaborators developed a program of linearization of such equations, they constructed transformations on appropriate Banach or Fréchet spaces which reduce the nonlinear PDEs to the equations given by their linear parts, and in this way solved the associated Cauchy problems, see [11] 12, 18] and references therein. The reducing transformations considered in this paper may be viewed as the quasi-linear counterpart of the linearization transformations. We hope that the analytic method of the program of linearization may give us a hint to the study of the above mentioned conjecture of Dubrovin.

Acknowledgments. The authors thank Boris Dubrovin and Daniel Sternheimer for helpful discussions and suggestive comments on the subject of the paper. This work is partially supported by NSFC No.10631050 and the National Basic Research Program of China (973 Program) No.2007CB814800.

\section{References}

[1] Camassa, R.; Holm, D. D. An integrable shallow water equation with peaked solitons. Phys. Rev. Lett. 71 (1993) 1661-1664. 
[2] Camassa, R.; Holm, D. D.; Hyman, J. M. A new integrable shallow water equation. Adv. Appl. Mech. 31 (1994), 1-33.

[3] Degasperis, A.; Procesi, M. Asymptotic integrability. in: Symmetry and perturbation theory (Rome, 1998), 23-37, World Sci. Publ., River Edge, NJ, 1999.

[4] Degasperis, A.; Holm, D. D.; Hone, A. N. I. A new integrable equation with peakon solutions. Teoret. Mat. Fiz. 133 (2002), 170-183. Translation in Theoret. and Math. Phys. 133 (2002), 1463-1474.

[5] Degiovanni, L.; Magri, F.; Sciacca, V. On deformation of Poisson manifolds of hydrodynamic type, Comm. Math. Phys. 253 (2005), 1-24.

[6] Drinfeld, V.; Sokolov, V. Lie algebras and equations of Korteweg-de Vries type, Journal of Mathematical Sciences 30 (1985), 1975 - 2036. Translated from Itogi Nauki i Tekhniki, Seriya Sovremennye Problemy Matematiki (Noveishie Dostizheniya) 24 (1984), 81-180.

[7] Dubrovin, B. On Hamiltonian perturbations of hyperbolic systems of conservation laws. II. Universality of critical behaviour. Comm. Math. Phys. 267 (2006), $117-139$.

[8] Dubrovin, B.; Liu, S.-Q.; Zhang, Y. On Hamiltonian perturbations of hyperbolic systems of conservation laws, I: Quasi-triviality of bi-Hamiltonian perturbations. Comm. Pure Appl. Math. 59 (2006), 559-615.

[9] Dubrovin, B.; Zhang, Y. Normal forms of integrable PDEs, Frobenius manifolds and Gromov-Witten invariants, Preprint arXiv: math.DG/0108160, 2001.

[10] Flato, M.; Lichnerowicz, A.; Sternheimer, D. Deformations of Poisson brackets, Dirac brackets and applications. J. Math. Phys. 17 (1976), 1754-1762.

[11] Flato, M.; Pinczon, G.; Simon, J. Non linear representations of Lie groups. Ann. Sci. École Norm. Sup. (4) 10 (1977), 405-418.

[12] Flato, M.; Simon, J.; Taflin, E. Asymptotic completeness, global existence and the infrared problem for the Maxwell-Dirac equations. Mem. Amer. Math. Soc. 127 (1997), no. 606.

[13] Fuchssteiner B.; Fokas A. S. Symplectic structures, their Bäcklund transformations and hereditary symmetries. Physica D 4 (1981) 47-66.

[14] Getzler, E. A Darboux theorem for Hamiltonian operators in the formal calculus of variations, Duke Math. J. 111 (2002), 535-560. 
[15] Lichnerowicz, A. Les varietes de Poisson et leurs algèbres de Lie associeés. J. Diff. Geom., 12 (1977), 253-300.

[16] Liu, S.-Q.; Zhang, Y. Deformations of semisimple bihamiltonian structures of hydrodynamic type. J. Geom. Phys. 54 (2005), 427-453.

[17] Liu, S.-Q.; Zhang, Y. On quasi-triviality and integrability of a class of scalar evolutionary PDEs. J. Geom. Phys. 57 (2006), 101-119.

[18] Taflin, E. Analytic linearization of the Korteweg-de Vries equation. Pacific J. Math. 108 (1983), 203-220. 